\title{
Estrategias Metodológicas Cognitivas En EI Desarrollo De La Destreza Auditiva En El Aprendizaje Del Idioma Inglés
}

\author{
Edison Renato Ruiz López \\ Magíster en Lingüística y didáctica de la enseñanza de Idiomas Extranjeros \\ Docente del Centro de Idiomas de la Escuela Superior Politécnica de \\ Chimborazo, Ecuador
}

Patricia Pilar Moyota Amaguaya

Magíster en Lingüística y didáctica de la enseñanza de Idiomas Extranjeros

Docente del Centro de Idiomas de la Escuela Superior Politécnica de

Chimborazo, Ecuador

Enrique Jesús Guambo Yerovi

Magister en Lingüística Aplicada al Aprendizaje del Idioma Inglés.

Docente de la Escuela Superior Politécnica de Chimborazo, Centro de Idiomas, Ecuador

Doi:10.19044/esj.2018.v14n17p250 URL:http://dx.doi.org/10.19044/esj.2018.v14n17p250

\begin{abstract}
This paper focuses on examining the application of cognitive methodological strategies and the effective development of listening skill in the English teaching process of the fourth level students room D and E of the Language Center of the National University of Chimborazo. This research had a quasi-experimental design. It is bibliographical, and it has a field and social intervention. The level of research is explanatory, descriptive, and correlational. The population under study comprised of 63 university students whom were divided into control and experimental groups. Taking into account the fact that there is an imbalance in the training of the English language skills within the execution of the educational process, it was made as a theoretical basis for the research. Later, a test and a post-test were applied to the two groups of students. Here, the application of cognitive methodological strategies was experimented in order to verify the relationship between the two variables. It was concluded that the mentioned strategies promote the development of the listening skill, considered by the writers as fundamental in the teaching of the languages.
\end{abstract}

Keywords: Listening Skill, Listening Strategies, Training, EFL Learners 


\section{Resumen}

El objetivo principal fue examinar la aplicación de estrategias metodológicas cognitivas y el desarrollo efectivo de la destreza auditiva en el proceso de enseñanza de inglés de los estudiantes de cuarto nivel paralelos "D" y "E" del Centro de Idiomas de la Universidad Nacional de Chimborazo. La investigación tuvo un diseño cuasi-experimental; fue de tipo bibliográfico, de campo y de intervención social. El nivel de investigación fue explicativo, descriptivo y correlacional. La población estudiada fueron 63 estudiantes universitarios quienes fueron divididos en un grupo de control y experimental. Teniendo en cuenta que, dentro de la ejecución del proceso educativo, existe un desequilibrio en la formación de las destrezas del idioma Inglés. Se comprobó que se construyó una base teórica para la investigación, $\mathrm{y}$ posterior se aplicó una prueba y un post-test a los dos grupos de estudiantes, donde se trabajó la aplicación de estrategias cognitivas metodológicas, para verificar la relación entre las dos variables. Se concluye que las mencionadas estrategias promueven el desarrollo de la destreza auditiva, considerada por los tratadistas como fundamental en la enseñanza de las lenguas.

Palabras Claves: destreza auditiva, instrucción de estrategias de escucha, estudiantes de EFL

\section{Introducción}

Si bien existen muchas publicaciones respecto a la formación de destrezas para la enseñanza del inglés, la mayor parte de investigaciones se orienta a las destrezas de escribir y leer, mientras que las de hablar y escuchar tienen menos impacto. Entre los trabajos encontrados se pueden mencionar los siguientes:

Cango (2012, p. xi) en su investigación de "Procesos cognitivos y factores motivacionales que inciden en el aprendizaje del Idioma Inglés en los cadetes del Tercer Año de Bachillerato del Colegio Militar T. Crn. Lauro Guerrero, en la Universidad Tecnológica Equinoccial en Loja-Ecuador", estableció objetivos específicos que precisan la incidencia de los procesos cognitivos en la enseñanza-aprendizaje del idioma inglés en los cadetes del tercer año de bachillerato a fin de determinar la incidencia de los factores motivacionales internos y externos que suceden en la enseñanza del idioma inglés en el aprendizaje de los estudiantes. Concluyó que “. Ningún proceso cognitivo que ocurre en la enseñanza del idioma inglés prevalece sobre otros; no obstante, se da un ligero predominio de la memorización, atención y concentración, que son necesarias para el aprendizaje del idioma, mas no es una práctica generalizada en los estudiantes. 
Tadeo (2010, p. vi) en su investigación "Estrategias Metodológicas en el desarrollo de la expresión oral en ingles de los estudiantes del noveno año de Educación Básica de la Unidad Educativa Teodoro Gómez De La Torre," plantea como objetivo el determinar de qué manera las Estrategias Metodológicas que utilizan los docentes desarrollan la expresión oral en inglés, posteriormente la identificación del nivel de desarrollo de la expresión oral en inglés de los estudiantes", en la cual se obtuvo que "los maestros no aplican estrategias adecuadas que permitan el buen desarrollo de la expresión oral, los maestros no motivan a sus estudiantes, los maestros continúan aplicando la metodología tradicional entre otras."

Las estrategias metodológicas cognitivas se definen como aquellas capacidades o competencias que se emplean con el estudiante a fin de guiar su atención, memorización, pensamiento y aprendizaje. Son el conjunto planificado y organizado de actividades orientadas a la construcción del conocimiento.

La enseñanza y aprendizaje del idioma inglés constituye un campo amplio de estudio, pues es un proceso donde se involucran aspectos cognitivos complejos y de diferentes niveles. De esta manera el término "estrategias" varía en su conceptualización, clasificación y sobre todo aplicación del modelo didáctico y al enfoque pedagógico planteado, lo que quiere decir que cada fundamentación varía dependiendo del punto de vista de cada investigador.

Zare (2012, p. 164) cita a Oxford (1990) y define a las estrategias de aprendizaje de una segunda lengua como: "acciones específicas tomadas por el alumno para hacer el aprendizaje fácil, rápido, agradable, eficaz y transferible a nuevas situaciones".

Zare (2012) cita a Brown (2007) el que menciona: "Las estrategias cognitivas están más limitadas a tareas específicas de aprendizaje e implican una manipulación más directa del material de estudio en sí". Por tanto, las estrategias cognitivas de aprendizaje orientadas al desarrollo de la destreza auditiva en la enseñanza del inglés pueden definirse como un conjunto de actividades dirigidas a interpretar el input de la escucha, lo que sirve de base para la adquisición efectiva de una lengua extranjera.

Para Gozález (2012, p. 1), "las estrategias de audición han sido definidas como técnicas o actividades que contribuyen a la comprensión y utilización de manera directa de información presentada de forma oral."

Renukadevi (2014), por otro lado, acota que las estrategias auditivas son técnicas o actividades que contribuyen directamente a evocar el input de la escucha. En los últimos días se han formulado una serie de estrategias de escucha que se adaptan a cada situación de escucha diferente y, por ello, en la enseñanza de la destreza auditiva, facilita a los estudiantes de idiomas a 
adaptar su comportamiento en la escucha para hacer frente a una variedad de situaciones, tipos de input y propósitos de la escucha.

Según Berne (2016) describe un enfoque basado en estrategias que cuando se enseña el input de una segunda lengua, los docentes deben alentar a los estudiantes a emplear las mismas estrategias que usan cuando escuchan en su lengua materna. Esto requiere llevar estas estrategias a un nivel consciente y ayudar a los estudiantes a aplicarlas en un contexto de la segunda lengua. El uso consciente de las estrategias permite controlar el uso de la estrategia y facilita su instrucción.

Citando a Baumann (2000, p. 94), “en este paso la responsabilidad de la adquisición de la destreza comienza a desplazarse al estudiante". En esta etapa el maestro inicia la actividad, pero solicita al estudiante que descifre el texto y empiece su trabajo independiente, pero aún se nota su presencia.

La responsabilidad de la ejecución del plan de estudios se va pasando progresivamente a los estudiantes, quienes finalmente deberán ser capaces de ejecutar la tarea de forma independiente.

Baumann (2000, p. 94) sostiene que "en el último paso, se da la transición de la responsabilidad del maestro en el aprendizaje a una responsabilidad compartida por el profesor y el alumno y finalmente a una responsabilidad total del estudiante."

Según Schuckermith (1987) citado por Morales (2012, pag. 10), "estas estrategias son procesos ejecutivos mediante los cuales se eligen, coordinan y aplican las habilidades. Se vinculan con el aprendizaje significativo y con el aprender a aprender."

Por consiguiente, las estrategias metodológicas cognitivas son un tipo de estrategias de aprendizaje importantes para la comprensión del inglés como idioma extranjero, ya que constituyen un apoyo necesario en la aplicación de procesos de aprendizajes y potencializan el desarrollo de la destreza auditiva de los estudiantes universitarios.

\section{Destreza Auditiva}

Escuchar no es lo mismo que oír. Mientras que la audición es un proceso fisiológico, escuchar es un proceso consciente que requiere estar mentalmente atentos. La escucha se ve como el anverso del hablar, y se conceptualiza como un proceso que implica la interpretación de mensajes que otros han transmitido intencionadamente. Cuando se visualiza de esta manera, la secuencia de escuchar, como la adquisición, procesamiento y retención de información en el contexto interpersonal, hace que la habilidad de escuchar para comprender implique la intención del remitente para poder formular una respuesta adecuada. Escuchar es esencial para la comunicación. Ser un buen oyente le ayuda a ver el mundo a través de los ojos de los demás, abriendo así su comprensión y aumentando su capacidad de empatía 
que es esencial para tener un buen nivel de desarrollo comunicativo. Sin embargo, tan simple como escuchar y reconocer a otras personas puede parecer, hacerlo bien, toma un esfuerzo sincero y mucha práctica.

Según estudios desarrollados por varios autores y sintetizados por Calvo (2008)," para los estudiantes de todos los niveles uno de las dificultades más frecuentes en el aprendizaje de una lengua extranjera es el relacionado con el proceso de la comprensión auditiva".

Saber una lengua no solo significa dominar su gramática y su lectura, sino también pronunciarla y para esto es necesario primero la percepción del sonido, la entonación, las pausas, la acentuación, las palabras, y las frases propias del idioma. Todos estos elementos le permiten al estudiante adquirir el sistema fonológico de la lengua y promover una respuesta.

Holec (1996) citado por Hurd (2008, pág. 99): sostiene que la integración refuerza la relevancia de las estrategias y proporciona las oportunidades regulares de uso que son necesarias para ser procesadas de manera significativa. La cuestión de la relevancia es también importante a un nivel más pragmático. Murphy (2005) citado por Hurd (2008, pág. 36) encontró en un estudio de estudiantes adultos de idiomas a distancia en la Open University (Reino Unido) que los alumnos son menos propensos a relacionarse con materiales o módulos que se perciben como un "extra" en lugar de parte de un programa de aprendizaje del lenguaje.

La formación de las microdestrezas de la escucha constituyen un conjunto integrado de habilidades aplicadas en conjunto con las estrategias que facilitan los contextos y situaciones comunicativas, esto se logra mediante la reflexión común para dotar al estudiante de herramientas que le permitan comprender que escuchar es más que saber el significado de las palabras y frases escuchadas.

Para hacer inferencias el oyente usa toda la información lingüística y no-lingüística a su disposición: para adivinar el sentido de lo que ha oído; para predecir lo que ha de suceder; o para completar lo que no ha oído (Schwartz, 1995, pág. 393).

Las investigaciones efectuadas para el desarrollo adecuado de las destrezas del idioma inglés se han concentrado en las destrezas de comprensión lectora, producción escrita y oral siendo relegada la destreza auditiva, a pesar de su importancia que en primer plano radica en la frecuencia de uso; es así que Rivers, Weaver, y Morley citados por González (2015, p. 95), consideran que en promedio se escucha dos veces más de lo que se habla, cuatro veces más de lo que se lee y cinco veces más de lo que se escribe. La destreza auditiva es presentada en varios ambientes educativos como un ejercicio simple e incierto para muchos estudiantes quienes son evaluados en base a sus habilidades para contestar preguntas acerca de un audio, pero en sí no se enseña como escuchar este idioma extranjero. 
De esta forma se ha establecido precedentes para validar la importancia del tema de investigación planteado, ya que los datos obtenidos sobre el uso de estrategias metodológicas en el Ecuador son muy escasos y de manera reiterada en el aprendizaje del idioma inglés o en el desarrollo de la destreza auditiva.

Frente a esta problemática y en la búsqueda de opciones para corregir las dificultades producidas por la insuficiente y en ocasiones nula explicación instructiva del desarrollo de la destreza auditiva en la enseñanza del inglés como idioma extranjero, se propuso la presente investigación, la cual se enfoca en la importancia de las estrategias metodológicas cognitivas como procedimiento en el desarrollo de la destreza de escucha de los estudiantes, que efectuada de una manera adecuada conducen hacia una escucha efectiva y consecuentemente la adquisición del idioma inglés.

\section{Metodología}

La investigación está enmarcada dentro del diseño cuasi experimental, debido a la imposibilidad de controlar totalmente el comportamiento de las variables de estudio, y por la imposibilidad de tener grupos de investigación aleatorios por el universo de trabajo conocido.

Para su empleo, es importante la recopilación direccionada de la información en grupos comparativos por medio de instrumentos aplicados en dos momentos, uno antes de la intervención y otro luego de la misma. El estudio será post facto, es decir posterior a la intervención con el fin de observar el cambio comportamental de los sujetos luego de la aplicación de estrategias metodológicas cognitivas en la enseñanza del inglés, con respecto al desarrollo de la destreza auditiva.

La investigación recurrió a información publicada tanto bibliográfica como documental, por medio de la recolección, selección, análisis y presentación de resultados sistematizados en publicaciones referentes a las estrategias metodológicas cognitivas alineadas con el constructivismo como modelo pedagógico referencial y la destreza auditiva del inglés.

Para el desarrollo de la investigación, se consideró tres criterios al momento de seleccionar el material:

- $\quad$ Pertinencia: Que significa que el material investigado deberá estar acorde con los objetivos trazados y aportar a ellos enfoques, teorías, conceptos, conocimientos y experiencias significativas que fundamenten su punto de vista.

- Exhaustividad: Es decir contar con todas las fuentes posibles y suficientes para sustentar el sistema de conocimientos que se defiende, sin excluir aquellas que tengan puntos de vista distintos a los expuestos. 
- $\quad$ Actualidad: El material empleado debe ser lo suficientemente actual para asegurar que la investigación esté alineada con los últimos estudios y avances.

La investigación fue de campo exploratorio, ya que fue desarrollada en los laboratorios de computo del Centro de Idiomas lugar en el que se recopiló la información de la aplicación de las estrategias metodológicas cognitivas en un entorno real.

La investigación tuvo intervención social, ya que el investigador planteó una propuesta de solución al problema investigado en el contexto donde se desarrolla la institución en la realizó el trabajo con todas sus características, problemática y fortalezas que en ella son determinantes.

La investigación permitió obtener la situación real en el comportamiento de las variables de estudio una vez que han sido confrontadas. En la investigación se experimentó la aplicación de estrategias metodológicas en el grupo experimental con el fin de verificar la relación con el desarrollo de la destreza auditiva.

La población de la investigación fueron los estudiantes del cuarto nivel de la Universidad Nacional del Chimborazo. Los estudiantes se caracterizaron por ser jóvenes adultos con un promedio de edad entre los 20 hasta los 22 años, de diferentes partes del Ecuador y diferentes clases sociales. Para obtener el número de estudiantes matriculados en cuarto nivel del idioma inglés, se tomará un cuadro estadístico realizado con la información de la Secretaría de la coordinación de inglés, en el cual se determinará el total de la población inmersa en la investigación que estará distribuida de la siguiente manera.

Tabla 1. Población investigada

\begin{tabular}{|c|c|c|c|c|c|c|}
\hline \multirow{2}{*}{ CURSOS } & \multicolumn{2}{|c|}{ Hombres } & \multicolumn{2}{|c|}{ Mujeres } & \multicolumn{2}{|c|}{ Total } \\
\hline & $\mathrm{N}^{\circ}$ & $\%$ & $\mathrm{~N}^{\circ}$ & $\%$ & $\mathrm{~N}^{\circ}$ & $\%$ \\
\hline 4 “E” & 17 & 56,67 & 13 & 43,33 & 30 & 100,00 \\
\hline 4 “D” & 8 & 24,24 & 25 & 75,76 & 33 & 100,00 \\
\hline \multicolumn{5}{|c|}{ TOTAL } & 63 & \\
\hline
\end{tabular}

Fuente: Secretaria Centro Idiomas UNACH

Elaborado por: Patricia Moyota

Debido a que los casos que componen el universo de trabajo son limitados, no hace falta la extracción de una muestra representativa, por lo que en la investigación se empleará el universo total de los casos registrados.

Las técnicas e instrumentos para la recolección de datos fueron los cuestionarios, que fueron aplicados en dos momentos Pre y Post test. El mismo que fue un instrumento de recolección de datos que definió la capacidad de medir efectivamente la variable que se estudia, 
Para el cálculo de validez, se sometió al test al criterio de expertos respecto al análisis de los ítems planteados para medir la destreza auditiva, entre ellos la delegada del Centro de Idiomas de la Facultad de Ciencias de la Salud de la Universidad Nacional de Chimborazo; Lic. Elizabeth Díaz, MsC, y el docente universitario; Lic. Hugo Romero $\mathrm{MsC}$, másteres en Lingüística Aplicada. Luego se aplicará el IVC (índice de validez del contenido)

Dónde:

$$
I V C=\frac{n e-\frac{N}{2}}{\frac{N}{2}}
$$

ne $=$ Número de expertos que consideran válido el ítem analizado

$\mathrm{N}=$ Número total de expertos consultados

Se validaron las preguntas del test y el pos test, a excepción de las preguntas 4,5 y 8 , las mismas que fueron revisadas y reformuladas tomando en cuenta el criterio de los expertos consultados.

Se define como confiabilidad al grado en que su aplicación repetida al mismo sujeto $\mathrm{u}$ objeto produce resultados iguales (consistentes $\mathrm{y}$ coherentes). Para validar el instrumento, se empleará el índice de confiabilidad de Crombach:

Dónde:

$$
\alpha_{e s t .}=\frac{k p}{1+p(k-1)}
$$

$\mathrm{k}=\mathrm{al}$ número de ítems del instrumento

$\mathrm{p}=$ Promedio de correlación lineal de los ítems calculado con la siguiente fórmula:

$\mathrm{P}=45$

$$
p=[k(k-1)] / 2
$$

$$
\begin{gathered}
\alpha_{\text {est. }}=\frac{10 * 45}{1+45(10-1)} \\
\alpha_{\text {est. }}=1,11
\end{gathered}
$$

Los valores del alfa de Crombach identifican la confiabilidad del test tomando en cuenta su estructuración, para eso se considera que los valores inferiores a 1 demuestran poca confiabilidad, mientras que, por el contrario, los valores superiores a 1 demuestran confiabilidad positiva, por tanto, el resultado obtenidos de 1,11 nos indica que el test y el pos test son confiables.

Para la aplicación del test se realizó un plan de intervención, donde inicialmente se aplicó el pre test a los estudiantes de cuarto nivel de inglés paralelos E y D del Centro de Idiomas de la Universidad Nacional de Chimborazo, con el fin de tener un punto de partida para la evaluación de la destreza auditiva. 
El paralelo "E" se considerará como población grupo de control, y el paralelo "D" como grupo experimental. El primer grupo (control), siguió su proceso de aprendizaje normalmente como se lo hace en dicha institución, mientras que en el grupo experimental se aplicación las estrategias listening for the gist y listening for detail durante 8 semanas, a razón de seis horas por semana, en horario de lunes, de $11 \mathrm{H} 00$ a $14 \mathrm{H} 00$ y miércoles de $13 \mathrm{H} 00$ a16H00.

Luego de las 8 semanas, se aplicó administró el pos test, con el que pretende evaluar las diferencias entre los grupos y determinar la eficiencia de las estrategias metodológicas cognitivas aplicadas.

Una vez aplicados las evaluaciones antes y después de la propuesta se realizará los siguientes pasos en el proceso de datos.

- $\quad$ Análisis crítico de los resultados.

- Tabulación de Resultados.

- Elaboración de Cuadros de Frecuencia y Porcentajes.

- $\quad$ Elaboración de Gráficos.

- En relación a las técnicas para el procesamiento y análisis de resultados de la hipótesis se comprobaron mediante el estadígrafo diferencia de medias. En el análisis de resultados se mostrará la incidencia de las estrategias metodológicas cognitivas en el desarrollo de la destreza auditiva, problema inició este estudio y que afecta a los estudiantes. Finalmente, se realizó una discusión teniendo en cuenta el marco teórico, la relación que existe entre las categorías, y los objetivos de esta investigación.

\section{Resultados}

Tanto el pre test como el pos test se han calificado sobre 10 puntos, y contienen la evaluación de las seis microdestrezas que definen la destreza auditiva: Identificación del input, Predicción, Idea principal, Ideas secundarias, Inferencia, Retención

Tabla 2. Análisis de promedios grupo control

\begin{tabular}{|c|c|c|}
\cline { 2 - 3 } \multicolumn{1}{c|}{} & PRE TEST & POS TEST \\
\hline Identificación del input (1) & 0,52 & 0,59 \\
\hline Predicción (2) & 0,89 & 0,97 \\
\hline Idea Principal (1) & 0,45 & 0,61 \\
\hline Ideas secundarias (3) & 0,94 & 0,97 \\
\hline Inferencias (2) & 0,73 & 0,88 \\
\hline Retención (1) & 0,39 & 0,39 \\
\hline Destreza Auditiva (10) & 3,92 & 4,29 \\
\hline
\end{tabular}

Fuente. Pre test y pos test grupo control

Elaborado por. Patricia Moyota 


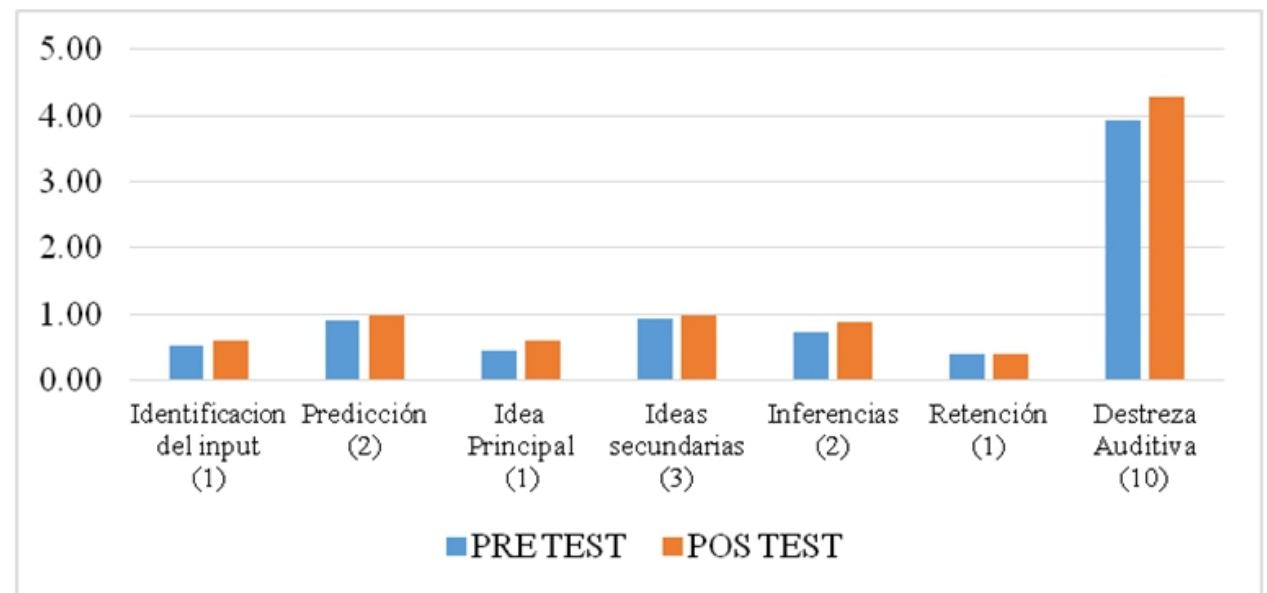

Gráfico 1. Análisis de promedios grupo control

Tabla 3. Análisis de promedios grupo experimental

\begin{tabular}{|c|c|c|}
\cline { 2 - 3 } \multicolumn{1}{c|}{} & PRE TEST & POS TEST \\
\hline Identificación del input (1) & 0,78 & 0,90 \\
\hline Predicción (2) & 1,34 & 1,59 \\
\hline Idea Principal (1) & 0,34 & 0,63 \\
\hline Ideas secundarias (3) & 1,2 & 2,23 \\
\hline Inferencias (2) & 0,4 & 0,97 \\
\hline Retención (1) & 0,3 & 0,67 \\
\hline Destreza Auditiva (10) & 4,365 & 6,99 \\
\hline
\end{tabular}

Fuente. Pre test y pos test grupo control

Elaborado por. Patricia Moyota

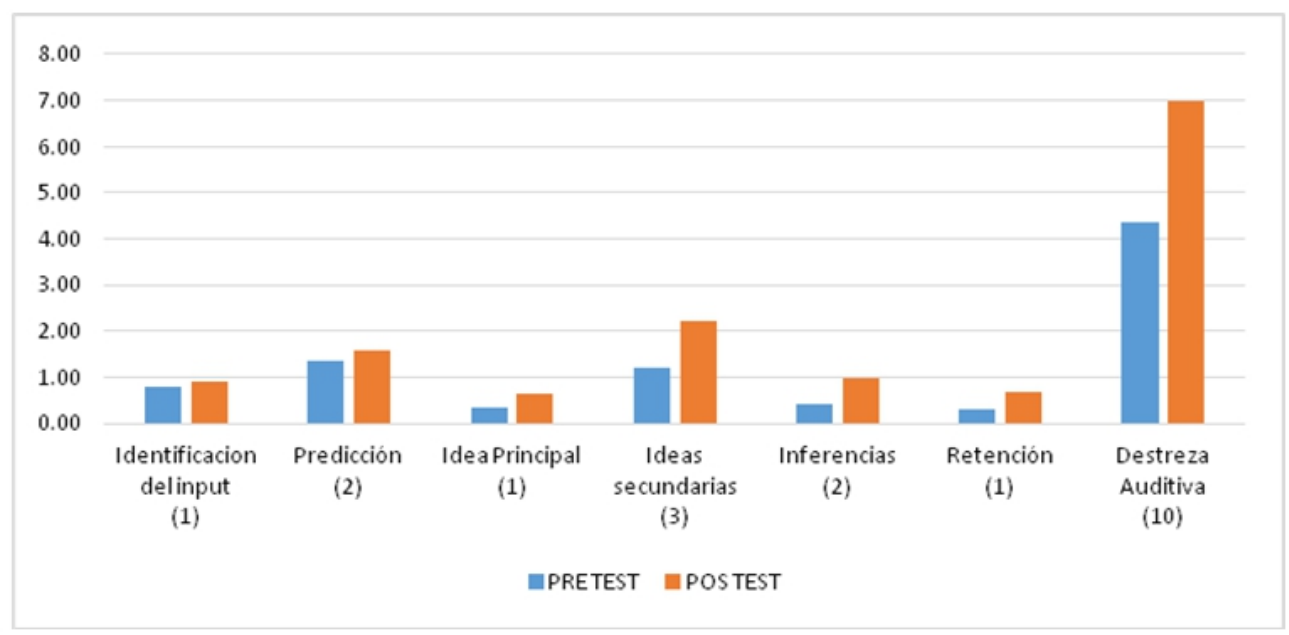

Gráfico 2. Análisis de promedios grupo experimental 


\section{Análisis De Resultados (Promedios)}

De los resultados obtenidos en la toma del pre test y el pos test se obtuvieron los siguientes resultados:

La puntuación en el caso del grupo de control inicialmente fue de 3,92 sobre 10 puntos en el pre test, mientras que en el pos test se elevó a 4,29 puntos sobre 10 puntos.

El mismo análisis en el grupo experimental arroja una calificación de 4,37 puntos sobre 10 , y se eleva a 6,99 sobre 10 en el pos test.

Tabla 4. Comparativo de promedios grupos control y experimental

\begin{tabular}{|c|c|c|}
\cline { 2 - 3 } \multicolumn{1}{c|}{} & G. CONT. & G. EXP. \\
\hline PRE TEST & 3,92 & 4,39 \\
\hline POST TEST & 4,29 & 6,99 \\
\hline VARIACIÓN & 8,62 & 37,20 \\
\hline
\end{tabular}

Fuente. Pre test y pos test grupo control

Elaborado por. Patricia Moyota

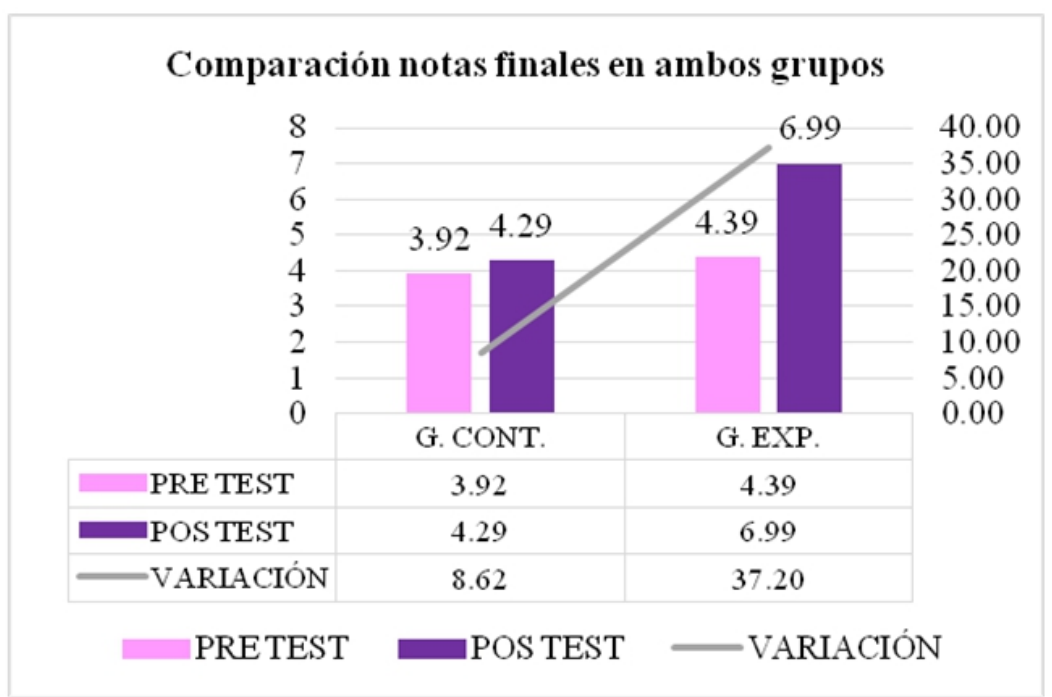

Gráfico 3. Comparativo de promedios grupo control y experimental

El análisis del grupo control evidencia un bajo nivel de desarrollo de los aspectos relacionados con la destreza auditiva que no mejora luego de las 8 semanas de trabajo, es decir, el estado inicial de las destrezas es bajo, y luego del tiempo de trabajo apenas mejora en menos de un punto.

Por el contrario, el grupo experimental inicia con una calificación en el pre test, y luego de la aplicación de las estrategias cognitivas durante las 8 semanas de trabajo, se elevó en más de dos puntos.

Lo anterior representa que el grupo control apenas tuvo un incremento de $8,62 \%$ entre ambas mediciones, mientras que el grupo experimental lo hizo en un $32,7 \%$. 


\section{Interpretación}

De acuerdo con los resultados obtenidos, la aplicación de estrategias metodológicas cognitivas tuvo una repercusión directa en los resultados del grupo experimental, esto es coincidente con el criterio vertido por (Zare, 2012, p. 164) cita a (Oxford, 1990): las estrategias metodológicas son "acciones específicas tomadas por el alumno para hacer el aprendizaje fácil, rápido, agradable, eficaz y transferible a nuevas situaciones".

\section{Análisis De Resultados (Dispersíon)}

Tabla 5. Comparativo de desviaciones estándar grupo control y experimental

\begin{tabular}{|c|c|c|}
\cline { 2 - 3 } \multicolumn{1}{c|}{} & G. CONT. & G. EXP. \\
\hline PRE TEST & 1,2 & 1,6 \\
\hline POS TEST & 0,99 & 1,36 \\
\hline VARIACIÓN & 21,21 & 17,65 \\
\hline
\end{tabular}

Fuente. Pre test y pos test grupo control

Elaborado por. Patricia Moyota

Tabla 6. Comparativo de varianzas grupo control y experimental

\begin{tabular}{|c|c|c|}
\cline { 2 - 3 } \multicolumn{1}{c|}{} & G. CONT. & G. EXP. \\
\hline PRE TEST & 1,44 & 2,57 \\
\hline POS TEST & 0,99 & 1,84 \\
\hline VARIACIÓN & 45,45 & 39,67 \\
\hline
\end{tabular}

Fuente. Pre test y pos test grupo control

Elaborado por. Patricia Moyota

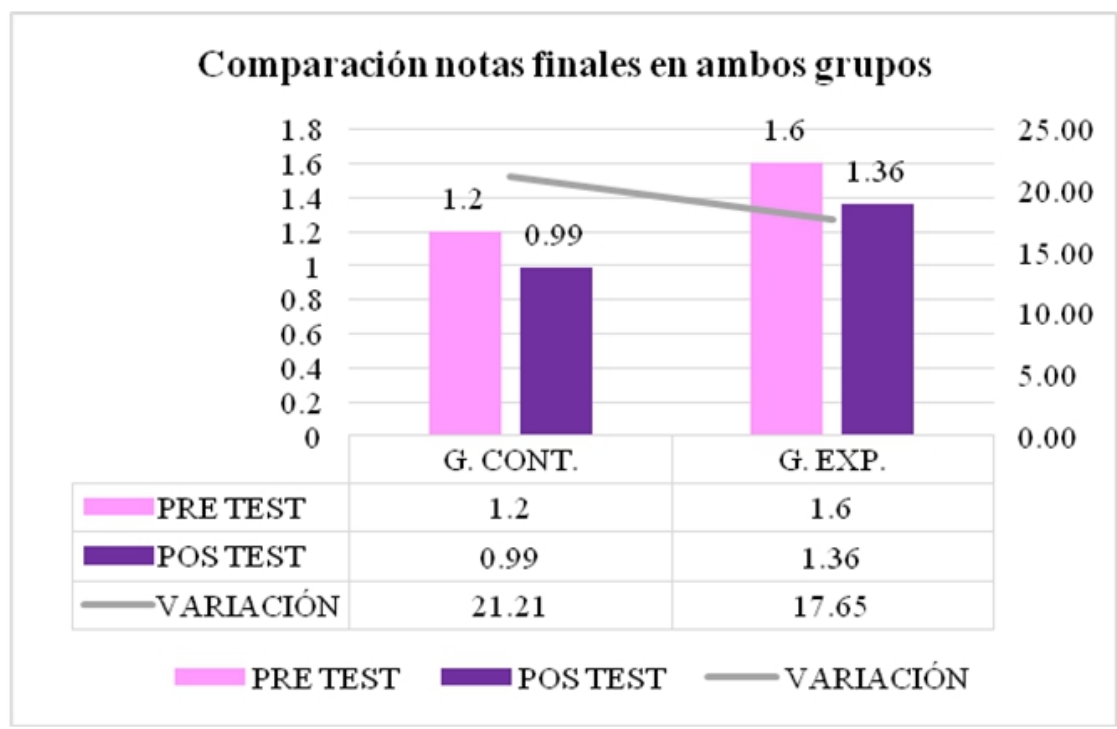

Gráfico 4. Comparativo de desviaciones estándar grupo control y experimental 


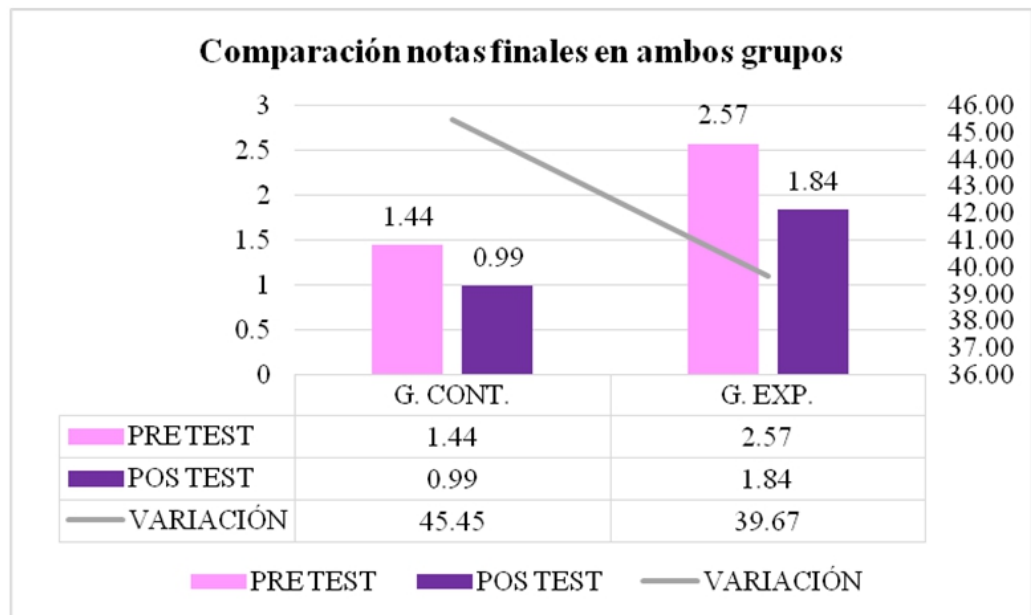

Gráfico 5. Comparativo de varianzas grupo control y experimental

De los resultados obtenidos, los valores de los estimadores estadísticos se incrementaron, pero en relación con la elevación de los puntajes en cada una de las tomas, sin embargo, en el estudio individual de cada grupo se notó que la desviación estándar baja porcentualmente $21,21 \%$ en el grupo control y $17,65 \%$ en el grupo experimental.

Por otro lado, la varianza tiene igual comportamiento, es decir una baja del 45,45\% en el grupo control y del 39,67\% en el grupo experimental.

Los decrementos en las medidas estadísticas de dispersión significan que existe menor dispersión en los resultados, es decir que existe más concentración de datos en cada uno de los grupos, pero con mayor impacto en el grupo experimental.

\section{Interpretación}

Lo anterior indica que además de incrementarse los promedios en cada uno de los grupos, pero con mayor impacto en el grupo experimental sometido a la aplicación de estrategias metodológicas cognitivas, los resultados experimentan menor grado de dispersión, o sea los estudiantes presentan menos diferencias en el desarrollo de la destreza auditiva.

De acuerdo con la teoría desarrollada, las estrategias metodológicas tienden a fomentar el trabajo mancomunado entre el grupo de estudiantes, por lo que los resultados son menos polarizados y tienden a concentrarse hacia la media, esta es la razón por la que disminuyen las medidas de dispersión, es necesario recordar que el ambiente favorable que generan, la estructuración secuencial del conocimiento, la motivación intrínseca y la participación activa hacen de las estrategias metodológicas una herramienta que permite afrontar en mejor forma las diferencias individuales y adaptar el conocimiento a las necesidades de cada estudiante. 


\section{Comprobación De La Hipótesis}

Tabla 7. Determinación de estimadores

\begin{tabular}{|c|c|c|}
\hline ESTIMADORES POS TEST & $\begin{array}{c}\text { GRUPO } \\
\text { CONTROL }\end{array}$ & GRUPO EXPERIMENTAL \\
\hline Promedio $(\mu)$ & 4,29 & 6,99 \\
\hline Varianza $(\mathrm{S} 2)$ & 0,99 & 1,84 \\
\hline Desviación Estándar $(\mathrm{s})$ & 0,99 & 1,36 \\
\hline Población $(\mathrm{N})$ & 33,0 & 30,00 \\
\hline
\end{tabular}

\section{Formulación de la hipótesis}

\section{Hipótesis nula ( $\mathrm{HO} \mu=\mu)$}

La aplicación de Estrategias Metodológicas Cognitivas no influye en el desarrollo de la destreza auditiva en los estudiantes de cuarto nivel de inglés del Centro de Idiomas Institucional de la Facultad de Ciencias de la Salud de la Universidad Nacional de Chimborazo.

Hipótesis alterna $(\mathrm{H} 1 \mu \neq \mu)$

La aplicación de Estrategias Metodológicas Cognitivas influye en el desarrollo de la destreza auditiva en los estudiantes de cuarto nivel de inglés del Centro de Idiomas Institucional de la Facultad de Ciencias de la Salud de la Universidad Nacional de Chimborazo.

Nivel de significación: 95\%

\section{Distribución muestral}

El valor correspondiente al $95 \%$ en puntuación tipificada $\mathrm{Z}$ es de $\pm 1,96$ tomado de niveles de confianza.

Tabla 8.

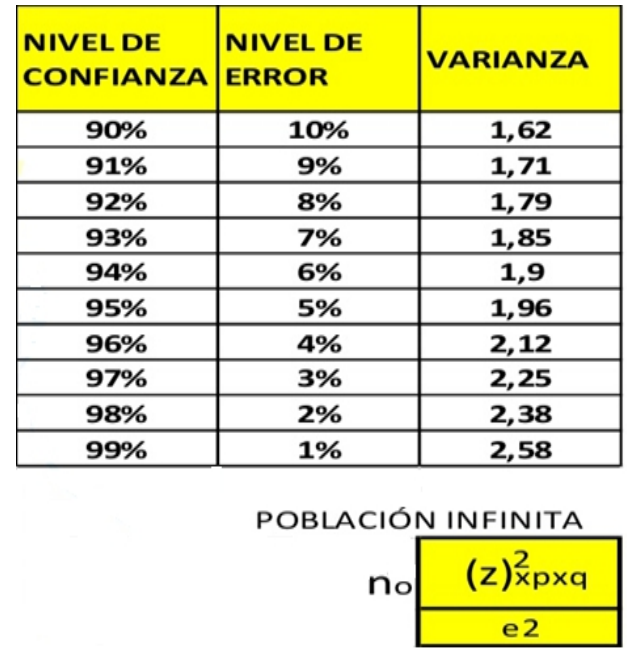


Determinación de la región de rechazo

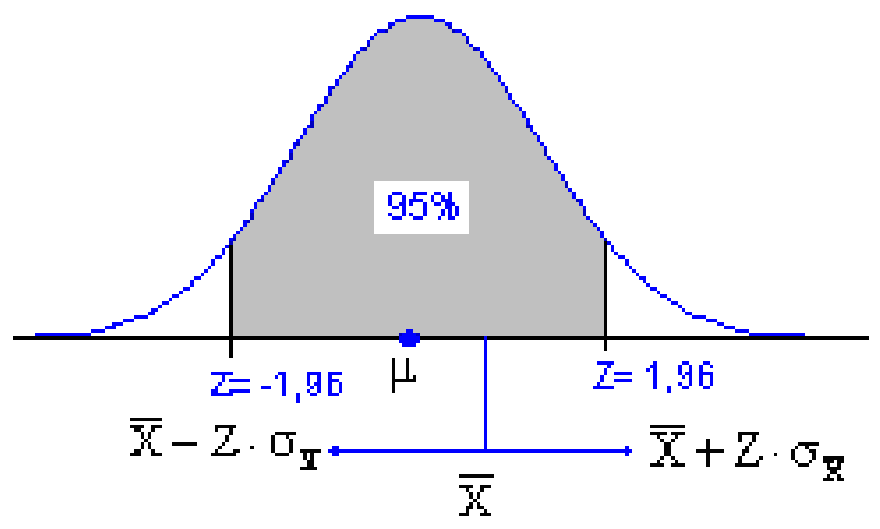

Cálculo estadístico

Error típico de medias muestrales

GRUPO CONTROL $=0,1758$

$$
S_{x}=\frac{s}{\sqrt{N-1}}
$$

GRUPO EXPERIMENTAL $=0,2518$

Cálculo del error típico de diferencias muestrales

$$
\begin{aligned}
& S_{d x}=\sqrt{s_{1}^{2}+s_{2}^{2}} \\
& S_{d x}=0,306073
\end{aligned}
$$

Determinación de la puntuación media tipificada "Z"

\section{Decisión}

$$
\begin{gathered}
Z=\frac{\mu_{1}+\mu_{2}}{S_{d x}} \\
Z=36,7
\end{gathered}
$$

Para un contraste bilateral a un nivel del $95 \%$ se tiene un valor teórico $\mathrm{Z}$ de $\pm 1,96$, es decir, se aceptaría la hipótesis nula con valores calculados dentro del intervalo entre $-1,96$ y $-1,96$. En vista que el valor calculado es superior a este intervalo, se rechaza la hipótesis nula y se acepta la hipótesis alterna, por cuanto las medias tipificadas de las dos muestras no son iguales, entonces La aplicación de Estrategias Metodológicas Cognitivas influye positivamente en el desarrollo de la destreza auditiva en los estudiantes de cuarto nivel de inglés del Centro de Idiomas Institucional de la Facultad de Ciencias de la Salud de la Universidad Nacional de Chimborazo. 


\section{Discusión De Resultados}

Los resultados obtenidos en la investigación de campo se caracterizan por demostrar que el empleo de estrategias metodológicas cognitivas es útil para el desarrollo de la destreza auditiva en el aprendizaje del inglés, aspecto en el que existe un acuerdo con los trabajos de investigación realizados y mencionados en la introducción de investigación, la diferencia radica en que en el caso de los trabajos citados hacen énfasis en destrezas de expresión oral, escrita y gramatical, mientras que el enfoque de esta investigación considera la destreza auditiva, que de acuerdo con tratadistas como (Córdova \& Coto, 2005, pág. 3) "Se podría decir que la destreza auditiva tiene tanta o más importancia que la destreza oral, dado que la una no funciona sin la otra, ya que hablar por el hecho de hablar, no constituye mayor mérito si lo que decimos no es recibido por otra persona".

En la actualidad, existe un acuerdo didáctico respecto al aprendizaje de una lengua. Con el estructuralismo lingüístico, se consideraba el desarrollo de las destrezas como un hecho horizontal, es decir todas en la misma dimensión y con la misma importancia, sin embargo, los resultados de aprendizaje obtenidos no son los esperados. Con el advenimiento de las nuevas teorías pedagógicas constructivistas y cognitivas, se ha establecido que el orden fundamental para aprender un idioma es escuchar, hablar, leer y escribir, se habla hoy de un bilingüismo aditivo, es decir de adoptar un cambio de código voluntario pero no sustractivo.

Los resultados obtenidos en la investigación nos indican que la apreciación anterior corrobora lo indicado antes, pues mientras que en el pre test la diferencia de promedios obtenidos es de 0,47 puntos sobre 10, en el pos test esta diferencia es de 2,7 puntos, y la dispersión medida por la desviación estándar es relativamente más baja en un 3,57\%.

Debe suponerse que el comportamiento del grupo experimental sometido a estrategias metodológicas cognitivas es la tónica general en el aprendizaje del inglés, ese es el aporte que se establece en este trabajo, sin embargo, dicha generalización es limitada por cuanto la población investigada es particular y no es completamente representativa de una realidad global, haría falta investigaciones similares en otros universos con el fin de poder comparar resultados y generar una teoría.

Otro aspecto importante que se debe destacar es que, metodológicamente hablando, los docentes y el sistema mismo, basan su acción educativa en el desarrollo de las cuatro principales destrezas de aprendizaje del idioma, incluida la auditiva, no es que no exista este aspecto formativo, el problema radica en que no está siendo desarrollada eficientemente tratada por el desconocimiento y no aplicación de estrategias metodológicas cognitivas. 
Luego de esta comprobación, y tomando como base la teoría desarrollada, se busca aportar con una propuesta que trate de solucionar el problema en el nivel investigado, para ello, se ha diseñado un Planning handout for listening for the gist and listening for details strategies implementation, con lo que se cumple lo propuesto en el objetivo general del trabajo.

Si bien la hipótesis se comprobó positivamente, y se determinó que la influencia de las estrategias metodológicas es determinante, fue interesante comprender que no todas las microdestrezas investigadas tuvieron el mismo comportamiento, por ejemplo, la comprensión de la idea principal, partió con una diferencia de promedios siendo mejor el grupo de control, y al final, no tuvo una diferencia significativa entre los dos grupos de estudiantes.

Cosa similar ocurrió con la inferencia, lo que nos indica que hay destrezas auditivas operacionales y conceptuales, estas últimas dependen del razonamiento del estudiante, por lo que son menos susceptibles al empleo de estrategias cognitivas, sería interesante profundizar en una investigación posterior esta diferencia que aportaría un elemento más de desarrollo para el aprendizaje del inglés.

\section{Conclusion}

La base estructural teórica dió sentido científico a la investigación y permitió la comparación con los resultados empíricos de campo, conocer la orientación que de acuerdo al modelo pedagógico constructivista debe darse a las estrategias metodológicas cognitivas es importante para aplicarlos en el desarrollo de la destreza auditiva en el aprendizaje del inglés.

El desarrollo de la destreza auditiva tiene un nivel muy bajo, sustentados en el pre test, en los estudiantes de idiomas del Centro de Idiomas de la UNACH, de acuerdo con la medición de sus microdestrezas, lo que se evidencia en el promedio obtenido en el pre test que apenas alcanza a 3,92 y 4,29 sobre 10 puntos en los grupos de control y experimental, respectivamente. Esto es sumamente grave tomando en cuenta que este tipo de destreza es fundamental para el verdadero aprendizaje de una lengua.

Si bien se nota un incremento en los puntajes en los dos grupos investigados: de control y experimental luego del período de clases de 8 semanas, este aumento es mayor en el grupo experimental, el mismo que tuvo la oportunidad de trabajar con estrategias metodológicas cognitivas. Esta aseveración se basa en los resultados del post test, los mismos que arrojan un incremento de 3,92 a 4,29 sobre 10 puntos equivalente a un $8,62 \%$, en el grupo de control; y, de 4,37 a 6,99 equivalente a $37,20 \%$, en el grupo experimental; es decir el incremento de promedio de medición de las microdestrezas es en el primero de apenas 0,37 puntos, mientras que en el segundo grupo es de 2,53 puntos sobre 10 . 
No todas las microdestrezas de la destreza auditiva tuvieron el mismo comportamiento si bien todas experimentaron un crecimiento, en ambos grupos, dos de ellas no dieron un contraste demasiado grande en cuanto a la diferencia entre grupos; la de determinación de la idea principal, en el grupo de control pasó de 0,45 a 0,61 sobre 10 puntos, y en el grupo experimental de 0,34 a 0,63 sobre 10 puntos, como puede verse la diferencia en el post test entre los dos grupos es de apenas 0,02 puntos sobre 10. Lo mismo sucede con la microdestreza inferencias que en el grupo de control pasó de 0,73 a 0,88 sobre 10 puntos y en el grupo experimental de 0,4 a 0,97 puntos, la diferencia de calificación del pos test entre grupos es de apenas 0,09 puntos. Se concluye que no todas las microdestrezas se desarrollan de la misma manera y en el mismo tiempo, algunas de ellas necesitan de la aplicación de estrategias metodológicas cognitivas, mientras que otras no lo requieren necesariamente.

\section{Recomendaciones}

Se recomienda a los docentes del Centro de Idiomas Institucional de la UNACH la aplicación de un sistema educativo que tome en cuenta que el desarrollo de la destreza auditiva es el inicio del proceso de aprendizaje de un idioma, lo que permite repetir para luego leer y escribir. Este orden lógico está sustentado en los procesos de adquisición del habla desarrollados por pedagogos y psicólogos educativos.

Luego de la aplicación del pos test, se nota la influencia de la aplicación de estrategias metodológicas cognitivas en el desarrollo de la destreza auditiva, por tanto, es necesario que esta herramienta se haga funcional y objetiva para el maestro.

De acuerdo con los resultados obtenidos con el fin de optimizar los recursos disponibles, se debe priorizar las microdestrezas de identificación del input, predicción, ideas secundarias, y retención, que son las más sensibles al empleo de estrategias metodológicas cognitivas.

\section{References:}

1. Baumann, J. (2000). La eficiencia de un modelo de instrucción directa en la enseñanza de la comprensión de ideas principales. Infancia y aprendizaje, 89-108.

2. Berne, J. (2016). Listening comprehension strategies: A review of the literature. Foreign Languaje annals, 512-533.

3. Calvo, D. (2008). Una propuesta pedagógica de uso de las tecnologías de la información para mejorar la comprensión auditiva en el aprendizaje del idioma inglés de las alumnas de educación, especialidad primaria del ISPP. Trujillo: Santo Tomás de Aquino. 
4. Cango, A. (2012). Procesos cognitivos y factores motivacionales que inciden en el aprendizaje del Idioma Inglés en los cadetes del Tercer Año de Bachillerato del Colegio Militar "Tcrn. Lauro Guerrero", período lectivo 2010-2011 . Loja: UTE.

5. Cango, A. (2012). Procesos cognitivos y factores motivacionales que inciden en el aprendizaje del Idioma Inglés en los cadetes del Tercer Año de Bachillerato del Colegio Militar T. Crn. Lauro Guerrero, en el año 2011. Loja - Ecuador: Universidad Tecnológica equinoccional. Obtenido de http://repositorio.ute.edu.ec/bitstream/123456789/12391/1/47932_1.p df

6. González, P. Y. (2015). EFL Teaching in the Amazon Region of Ecuador: A Focus on. Canadian Center of Science and Education, 94103.

7. Gozálz, A. Y. (2012). Las estretegias de audición y lectura en el aprendizaje de la lengua inglesa. Una sistematizacíon teórica. Ciencias Pedagógicas.

8. Hurd, S. Y. (2008). Languaje learning strategies in independnet settings. Great Britain: Multilingual Matters.

9. Renukadevi, D. (2014). The Role of Listening in Language Acquisition; the Challenges \& Strategies in Teaching Listening . International Journal of Education and Information Studies., 59-64.

10. Schwartz, A. (1995). LA ENSEÑANZA DE ESTRATEGIAS DE APRENDIZAJE. Centro Virtual Cervantes, 393-394.

11. Tadeo, C. C. L., \& Tadeo, C. Z. E. (2010). Estrategias Metodológicas en el desarrollo de la expresión oral en ingles de los estudiantes del noveno año de Educación Básica de la Unidad Educativa Teodoro Gómez De La Torre. Licenciatura, Licenciatura en ciencia de la Educación, especialidad Inglés. Ibarra - Ecuador: Universidad del Norte.

12. Tadeo, C. (2010). Estrategias metodológicas en el desarrollo de la expresión oralen inglés de los estudiantes del noveno año de Educación Básica de la Unidad Educativa Teodoro Gómes de la Torre. Ibarra: Universidad Técnica del Norte.

13. Zare, P. (2012). Language Learning Strategies Among EFL/ESL Learners: A Review of Literature. International Journal of Humanities and Social Science, 162-169. 